Dev Neurosci 2017;39:207-214

DOI: $10.1159 / 000454856$
Received: September 5, 2016

Accepted after revision: November 30, 2016

Published online: January 18, 2017

\title{
Diffusion Tensor Imaging Detects Occult Cerebellar Injury in Severe Neonatal Hypoxic-Ischemic Encephalopathy
}

\author{
Monica E. Lemmon ${ }^{a-c}$ Matthias W. Wagner ${ }^{c, d, i}$ Thangamadhan Bosemani ${ }^{c, d}$ \\ Kathryn A. Carson ${ }^{e, g}$ Frances J. Northington ${ }^{c, f}$ Thierry A.G.M. Huisman ${ }^{c, d}$ \\ Andrea Porettic, d, h \\ a Division of Pediatric Neurology, Department of Pediatrics, Duke University School of Medicine, Durham, NC, \\ ${ }^{b}$ Division of Pediatric Neurology, Department of Neurology, ' Neuro Intensive Care Nursery Group, \\ d Section of Pediatric Neuroradiology, Division of Pediatric Radiology, The Russell H. Morgan Department of \\ Radiology and Radiological Science, Charlotte R. Bloomberg Children's Center, e'Division of General Internal \\ Medicine, Department of Medicine, and ${ }^{\mathrm{f}}$ Division of Neonatology, Department of Pediatrics, The Johns Hopkins \\ University School of Medicine, ${ }^{9}$ Department of Epidemiology, The Johns Hopkins Bloomberg School of Public \\ Health, and hepartment of Neurogenetics, Kennedy Krieger Institute, Baltimore, MD, USA; 'Department of \\ Diagnostic and Interventional Radiology, University Hospital of Zurich, Zurich, Switzerland
}

\section{Keywords}

Hypoxic-ischemic encephalopathy - Neonatal cerebellum . Magnetic resonance imaging . Diffusion tensor imaging

\begin{abstract}
Background: Despite the benefits of whole-body hypothermia therapy, many infants with hypoxic-ischemic encephalopathy (HIE) die or have significant long-term neurodevelopmental impairment. Prospectively identifying neonates at risk of poor outcome is essential but not straightforward. The cerebellum is not classically considered to be a brain region vulnerable to hypoxic-ischemic insults; recent literature suggests, however, that the cerebellum may be involved in neonatal HIE. In this study, we aimed to assess the microstructural integrity of cerebellar and linked supratentorial structures in neonates with HIE compared to neurologically healthy neonatal controls. Methods: In this prospective co-
\end{abstract}

\section{KARGER}

(C) 2017 S. Karger AG, Basel

E-Mail karger@karger.com

www.karger.com/dne hort study, we performed a quantitative diffusion tensor imaging (DTI) analysis of the structural pathways of connectivity, which may be affected in neonatal cerebellar injury by measuring fractional anisotropy (FA) and mean diffusivity (MD) within the superior, middle, and inferior cerebellar peduncles, dentate nuclei, and thalami. All magnetic resonance imaging (MRI) studies were grouped into 4 categories of severity based on a qualitative evaluation of conventional and advanced MRI sequences. Multivariable linear regression analysis of cerebellar scalars of patients and controls was performed, controlling for gestational age, age at the time of MRI, and HIE severity. Spearman rank correlation was performed to correlate DTI scalars of the cerebellum and thalami. Results: Fifty-seven (23 females, 40\%) neonates with HIE and 12 (6 females, 50\%) neonatal controls were included. There were 8 patients (14\%) in HIE severity groups 3 and 4 (injury of the basal ganglia/thalamus and/or cortex). Based on a qualitative analysis of conventional and DTI im-

Andrea Poretti, MD

The Johns Hopkins University School of Medicine

Charlotte R. Bloomberg Children's Center, Sheikh Zayed Tower, Room 4174

1800 Orleans Street, Baltimore, MD 21287-0842 (USA)

E-Mail aporett1@jhmi.edu 
ages, no patients had evidence of cerebellar injury. No significant differences between patients and controls were found in the FA and MD scalars. However, FA values of the middle cerebellar peduncles $(0.294$ vs. $0.380, p<0.001)$ and $M D$ values of the superior cerebellar peduncles $(0.920 \mathrm{vs}$. $1.007 \times 10^{-3} \mathrm{~mm} / \mathrm{s}^{2}, p=0.001$ ) were significantly lower in patients with evidence of moderate or severe injury on MRI (categories 3 and 4) than in controls. In patients, cerebellar DTI scalars correlated positively with DTI scalars within the thalami. Conclusion: Our results suggest that infants with moderate-to-severe HIE may have occult injury of cerebellar white-matter tracts, which is not detectable by the qualitative analysis of neuroimaging data alone. Cerebellar DTI scalars correlate with thalamic measures, highlighting that cerebellar injury is unlikely to occur in isolation and may reflect the severity of HIE. The impact of concomitant cerebellar injury in HIE on long-term neurodevelopmental outcome warrants further study.

(c) 2017 S. Karger AG, Basel

\section{Introduction}

Hypoxic-ischemic encephalopathy (HIE) occurs in approximately 1-3 newborns per 1,000 live full-term births and is a major cause of neonatal death and neurodevelopmental disability [1]. Whole-body therapeutic hypothermia is widely recognized as one of the few effective treatments for HIE to mitigate secondary injury [2]. However, approximately $45 \%$ of infants with HIE die or have significant neurodevelopmental disability $[3,4]$. Prospectively identifying which infants will have poor outcomes is not straightforward and reliable objective predictors are needed [5].

Magnetic resonance imaging (MRI) is the neuroimaging technique of choice in the diagnostic work-up of neonatal HIE. Conventional MRI sequences may show lesions 1-2 days after injury. These findings gradually become more conspicuous within the first week after birth [6]. Conventional MRI sequences, however, may underestimate the extent of injury, particularly in the first few days after HIE [7]. In addition, the ability of conventional MRI to predict neurodevelopmental outcome is limited [8-10], and up to one-quarter of patients with an apparently normal MRI study will have moderate or severe long-term developmental delay [11]. Advanced MRI sequences such as diffusion tensor imaging (DTI) provide additional valuable information for the diagnosis and prognostic outcome of neonatal HIE $[12,13]$. The early identification of asphyxiated neonates at risk for poor neurodevelopmental outcome is critical to ensure targeted treatments, follow-up, and appropriate intervention.

Four topographic neuroimaging patterns of brain injury have been delineated in neonatal HIE including cerebral cortex, subcortical and central white matter, deep nuclear structures (especially the putamen and thalamus), and brainstem tegmentum [14]. Correlations have been shown between these distinct imaging patterns of injury and severity, temporal characteristics of hypoxicischemic insults, and neurodevelopmental outcome [14]. The cerebellum is not classically considered a brain region vulnerable to hypoxic-ischemic insults, and the literature about cerebellar involvement in neonatal HIE is consequently limited. However, recent data have suggested cerebellar injury in neonatal HIE. Alderliesten et al. [15] found neuropathological evidence of cerebellar injury in 19 of 23 (83\%) neonates with HIE who died; much of this injury was not evident on qualitative evaluation of conventional and advanced MRI sequences. In a cohort of 172 asphyxiated neonates, advanced neuroimaging techniques increased the detection rate of cerebellar involvement in neonatal HIE which was identifiable on matching conventional MRI sequences in only $4 \%$ of the cases [16].

In this prospective cohort study, we analyzed cerebellar DTI data in newborns with HIE treated with therapeutic whole-body hypothermia and in neonatal control patients, in order to assess the microstructural integrity of various cerebellar structures and structurally linked brainstem and supratentorial structures.

\section{Material and Methods}

This study was approved by the Johns Hopkins Institutional Review Board and a consent waiver was obtained.

\section{Patients and Controls}

The inclusion criteria for the neonates were (1) a diagnosis of HIE and fulfillment of criteria for therapeutic hypothermia [17] and (2) the availability of DTI data without artifacts enabling highquality post-processing. Exclusion criteria included (1) the discontinuation of therapeutic hypothermia prior to $72 \mathrm{~h},(2)$ an alternative or additional diagnosis based on conventional brain MRI studies, and (3) the presence of cerebellar injury on qualitative MRI assessment. Patients were prospectively enrolled in an ongoing research cohort that included all neonates with HIE who underwent therapeutic hypothermia between 1 January 2010 and 31 December 2014, in the Johns Hopkins Neuro Intensive Care Nursery. Neonatal controls were selected from our pediatric MRI database, which includes all children at our institution with brain MRI and DTI sequences between September 2010 and 31 December 2014 (approx. 10,000 DTI studies). The following inclusion criteria were 

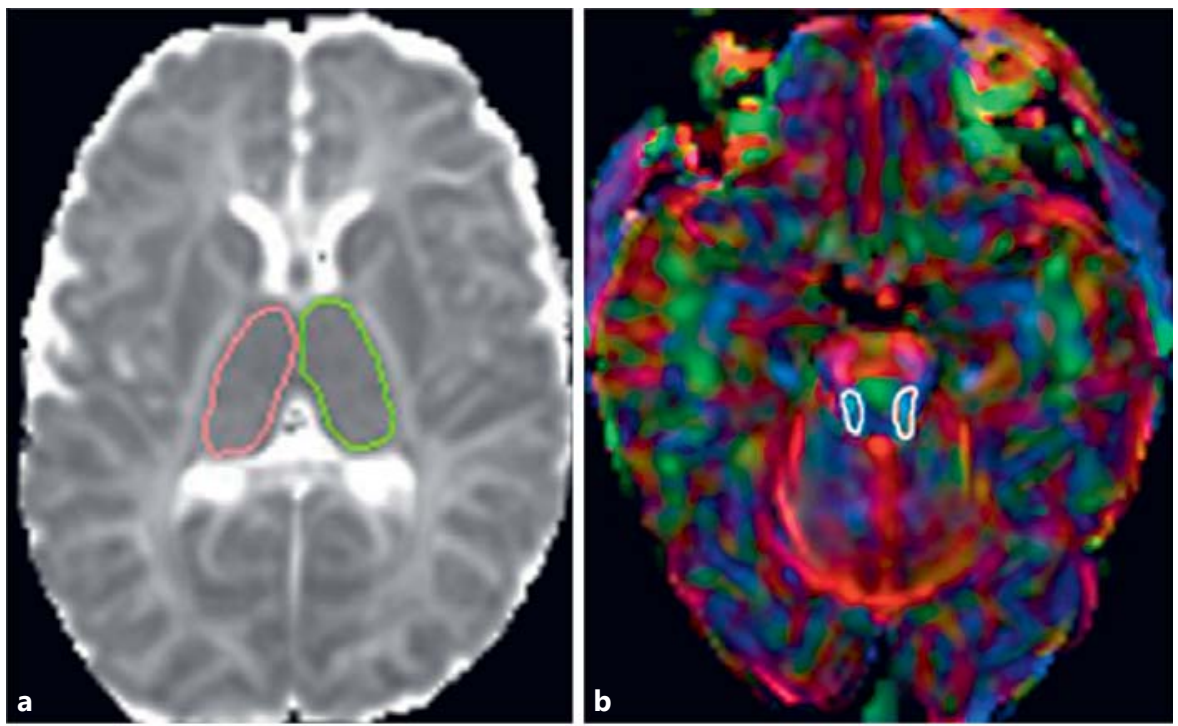

Fig. 1. a Axial trace of diffusion map shows the positioning of the regions of interest (ROIs) covering the bilateral thalami. bd Axial color-coded fractional anisotropy maps show the positioning of the ROIs within the bilateral superior cerebellar peduncles (b), dentate nuclei (c), and middle and inferior cerebellar peduncles (d) in 1 representative neonate with hypoxic-ischemic encephalopathy and hypothermia therapy.
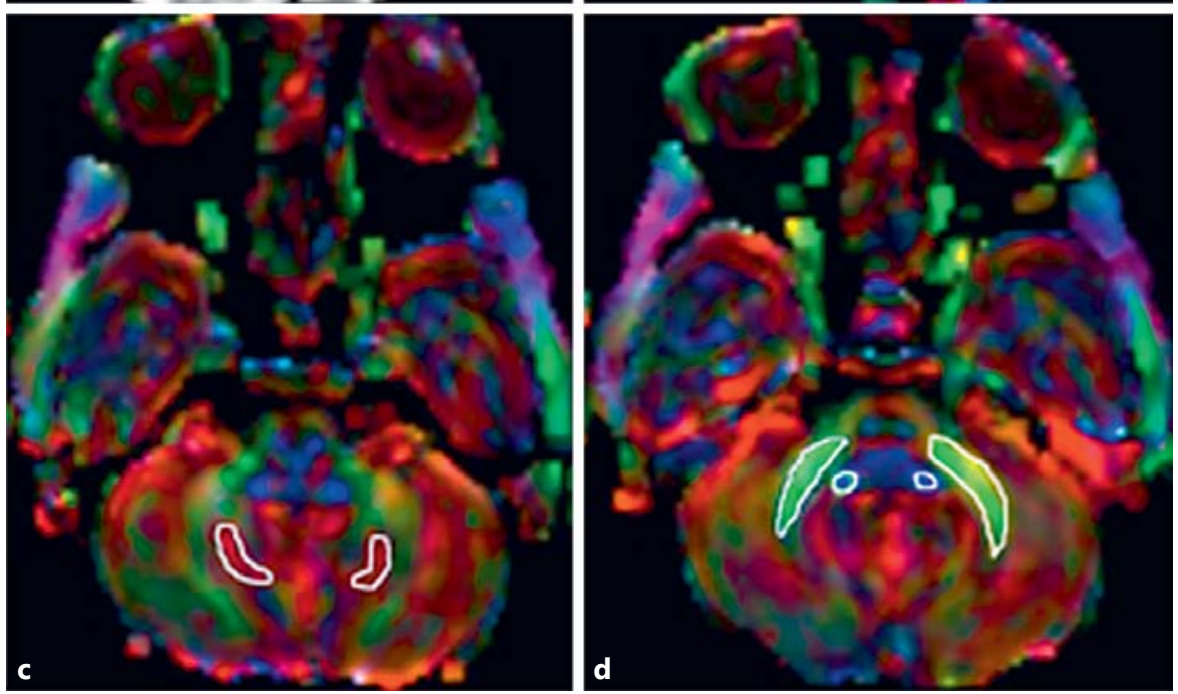

used: (1) an age at MRI of between 1 and 28 days, (2) a gestational age at birth of between 36 and 41 weeks, (3) normal brain anatomy, (4) an absence of known or suspected neurological disorders, and (5) the availability of DTI raw data without artifacts, thus enabling high-quality post-processing. All neonatal controls who met the inclusion criteria were included in this study. Brain MRI, including DTI data of the neonatal controls, was performed for clinical indications, such as facial skin lesions or evaluation of soft-tissue pathologies confined to the head and neck region.

Demographic data and clinical information were prospectively collected and included maternal and birth history, Apgar score, and cord-blood gas measurements ( $\mathrm{pH}$ and base excess).

\section{Acquisition of MRI Data}

All brain MRI studies were performed on a 1.5-tesla clinical scanner (Siemens, Erlangen, Germany) and included isotropic three-dimensional T1-weighted and axial T2-weighted images, susceptibility-weighted imaging, and a single-shot spin echo, echo planar axial DTI sequence with diffusion gradients along 20 noncollinear directions (effective high $b$ value of $1,000 \mathrm{~s} / \mathrm{mm}^{2}$ ). We performed an additional measurement without diffusion weighting $\left(b=0 \mathrm{~s} / \mathrm{mm}^{2}\right)$. For the acquisition of DTI, the following parameters were used: repetition time $8,500 \mathrm{~ms}$, echo time $86 \mathrm{~ms}$, slice thickness $2.0 \mathrm{~mm}$, field of view $240 \times 240 \mathrm{~mm}$, and matrix size 192 $\times 192$. A parallel-imaging integrated parallel acquisition technique 2 with generalized autocalibrating partial parallel acquisition reconstruction was used. The acquisition was repeated twice to enhance the signal-to-noise ratio. Imaging was typically performed with the advantage of an MRI-compatible incubator (LMT, Germany). The DTI acquisition protocol was assessed weekly and was stable throughout the study period.

\section{Qualitative MRI Analysis}

All patients were grouped into 4 degrees of HIE severity based on conventional and advanced (trace of diffusion and apparent diffusion coefficient [ADC] maps) sequences by a pediatric neuro- 
Table 1. Demographic and clinical characteristics of neonates with HIE and hypothermia therapy and controls

\begin{tabular}{lcl}
\hline Characteristics & HIE $(n=57)$ & Controls $(n=12)$ \\
\hline Females, $n$ & $23(40 \%)$ & $6(50 \%)$ \\
Gestational age, weeks & $39(35-42)$ & $39(36-41)$ \\
5-min Apgar score & $4(0-9)$ & $9(4-9)$ \\
10-min Apgar score & $6(2-10)$ & n.a. \\
Cord pH & $6.96(6.60-7.44)$ & n.a. \\
Base excess, mmol/L & $15(5-30)$ & n.a. \\
\hline
\end{tabular}

Values denote median (range), unless otherwise indicated. HIE, hypoxic-ischemic encephalopathy; n.a., not available.

radiologist (T.B.) and a pediatric neurologist (A.P.) in consensus: (1) normal, (2) injury of the periventricular white matter (T2-hyperintense signal or restricted diffusion) $=$ mild injury, (3) injury of the basal ganglia/thalamus or cortex $=$ moderate injury, and (4) injury of the basal ganglia/thalami and cortex = severe injury (modified from Barkovich et al. [18]). In addition, all studies were qualitatively assessed for the presence of cerebellar injury (focal ischemia, hemorrhage, or edema).

\section{Quantitative DTI Analysis}

DTI raw data were transferred to an off-line workstation for further post-processing. DTIStudio, DiffeoMap, and ROIEditor software were used. The raw DTI data were first coregistered to at least 1 image and corrected for eddy current and subject motion using a 12-mode affine transformation. The following maps were generated: fractional anisotropy (FA), color-coded FA, and trace of diffusion. Regions of interest (ROIs) were manually drawn, covering the bilateral thalami, superior (SCP), middle (MCP), and inferior (ICP) cerebellar peduncles, and the dentate nucleus using axial trace of diffusion and color-coded FA maps (Fig. 1). For each structure analyzed, 3 different ROIs on contiguous slices were placed, and the mean FA and trace-of-diffusion values were calculated. ROIs were placed manually by 1 blinded study team member (M.W.W.) and confirmed by a second blinded study team member (A.P.). Mean diffusivity (MD) values were subsequently calculated using the following formula: $\mathrm{MD}=$ trace of diffusion $/ 3$.

\section{Statistical Analysis}

First, paired $t$ tests were used to test for differences between the right and left side in the FA and MD values of the thalami, SCP, MCP, ICP, and dentate nuclei. No differences were found. Therefore, averages of the right and left values were used for further analysis. In order to control for gestational age, age at the time of MRI, and MRI category, multivariable linear regression analysis of cerebellar DTI scalars of all neonates with HIE and hypothermia and neonatal controls was performed. A subgroup analysis of neonates with severe HIE and hypothermia (categories 3 and 4 based on the qualitative analysis) and neonatal controls, which was not adjusted for MRI category, was also performed. The Spearman rank correlation was performed to correlate cerebellar DTI scalars with DTI scalars of the thalami, partialling out gestational age, age at MRI, and MRI category. Analyses were performed using SAS software v9.3 (SAS Institute, Inc., Cary, NC, USA). All tests were 2-sided, and observed differences were considered statistically significant when $p<0.05$. No adjustment was made for multiple comparisons. The statistical analysis was performed by a certified statistician (K.A.C.).

\section{Results}

\section{Patients and Controls}

Between 1 January 2010 and 31 December 2014, 125 neonates with HIE underwent therapeutic whole-body hypothermia treatment in our tertiary pediatric hospital. Sixty-eight neonates were excluded due to lacking DTI data $(n=65)$, significant artifacts $(n=1)$, or the premature discontinuation of hypothermia $(n=2)$. Fifty-seven fulfilled the inclusion criteria for this study (23 females), and the median gestational age was 39 weeks (range 3542 weeks). Twelve neonatal control patients were eligible for inclusion. Brain MRIs of the patients were performed at a median age of 9 days (range 4-15 days); brain MRIs of the controls were performed at a median age of 7.5 days (range 1-15 days). Characteristics of patients and controls are shown in Table 1.

\section{Qualitative MRI Analysis}

The MRI studies of the neonates with HIE and hypothermia were grouped as following: 11 (19\%) in category 1 (normal), 38 (67\%) in category 2 (mild injury), 3 (5\%) in category 3 (moderate injury), and 5 (9\%) in category 4 (severe injury). No patients had evidence of cerebellar injury, based on a qualitative analysis of conventional and DTI images. The MRI category for all controls was 0 .

\section{DTI Analysis}

The cerebellar DTI scalars for the patients and controls are summarized and compared in Table 2. There were no significant differences in FA and MD between HIE patients and neonatal controls for the studied anatomical structures. However, there were significant differences in FA of the MCP $(0.294$ vs. $0.380, p<0.001)$ and MD of the SCP $\left(0.920\right.$ vs. $\left.1.007 \times 10^{-3} \mathrm{~mm} / \mathrm{s}^{2}, p=0.001\right)$ between the subgroup of patients with moderate-to-severe HIE (categories 3 and 4 based on a qualitative analysis of the MRI studies) and neonatal controls (Table 3).

Patients' median values of FA and MD of the thalami were 0.166 (range $0.114-0.230$ ) and $1.039 \times 10^{-3} \mathrm{~mm} / \mathrm{s}^{2}$ (range $0.704-1.389 \times 10^{-3} \mathrm{~mm} / \mathrm{s}^{2}$ ), respectively. The results of the correlation between thalamic and cerebellar involvement are shown in Table 4. There were significant
210

Dev Neurosci 2017;39:207-214

DOI: $10.1159 / 000454856$
Lemmon/Wagner/Bosemani/Carson/

Northington/Huisman/Poretti 
Table 2. Differences in cerebellar DTI scalars between 57 neonates with HIE and hypothermia therapy and 12 neonatal controls

\begin{tabular}{lllll}
\hline DTI scalars & Anatomical region & HIE, mean $(95 \% \mathrm{CI})$ & Controls, mean $(95 \% \mathrm{CI})$ & $p$ value \\
\hline FA & SCP & $0.337(0.324-0.350)$ & $0.325(0.298-0.351)$ & 0.38 \\
& MCP & $0.317(0.300-0.333)$ & $0.339(0.305-0.372)$ & 0.21 \\
& ICP & $0.267(0.247-0.287)$ & $0.296(0.255-0.336)$ & 0.17 \\
& Dentate nuclei & $0.143(0.131-0.156)$ & $0.149(0.124-0.174)$ & 0.64 \\
\hline $\mathrm{MD}, \times 10^{-3} \mathrm{~mm} / \mathrm{s}^{2}$ & SCP & $0.970(0.933-1.007)$ & $0.994(0.919-1.070)$ & 0.53 \\
& MCP & $1.109(1.076-1.142)$ & $1.074(1.008-1.141)$ & 0.31 \\
& ICP & $0.995(0.948-1.041)$ & $0.991(0.897-1.085)$ & 0.94 \\
& Dentate nuclei & $1.185(1.144-1.227)$ & $1.232(1.147-1.317)$ & 0.28 \\
\hline
\end{tabular}

DTI scalars have been adjusted for gestational age, age at the time of MRI, and MRI category by linear regression; least-squares means are presented. DTI, diffusion tensor imaging; FA, fractional anisotropy; HIE, hypoxic-ischemic encephalopathy; ICP, inferior cerebellar peduncle; MCP, middle cerebellar peduncle; MD, mean diffusivity; SCP, superior cerebellar peduncle.

Table 3. Differences in cerebellar DTI scalars between 8 neonates with severe HIE and hypothermia therapy and 12 neonatal controls

\begin{tabular}{llllc}
\hline DTI scalars & Anatomical region & HIE, mean $(95 \% \mathrm{CI})$ & Controls, mean (95\% CI) & $p$ value \\
\hline FA & SCP & $0.347(0.319-0.374)$ & $0.328(0.306-0.350)$ & 0.28 \\
& MCP & $0.294(0.259-0.328)$ & $0.380(0.352-0.408)$ & $<\mathbf{0 . 0 0 1}$ \\
& ICP & $0.277(0.237-0.316)$ & $0.289(0.258-0.321)$ & 0.60 \\
& Dentate nuclei & $0.140(0.118-0.161)$ & $0.156(0.138-0.173)$ & 0.24 \\
\hline $\mathrm{MD}, \times 10^{-3} \mathrm{~mm} / \mathrm{s}^{2}$ & SCP & $0.920(0.884-0.956)$ & $1.007(0.977-1.036)$ & $\mathbf{0 . 0 0 1}$ \\
& MCP & $1.106(1.061-1.151)$ & $1.090(1.053-1.127)$ & 0.57 \\
& ICP & $0.988(0.944-1.033)$ & $0.981(0.945-1.017)$ & 0.78 \\
& Dentate nuclei & $1.184(1.083-1.285)$ & $1.199(1.117-1.281)$ & 0.82 \\
\hline
\end{tabular}

Statistically significant differences are in bold type. DTI scalars have been adjusted for gestational age and age at the time of MRI by linear regression; least-squares means are presented. DTI, diffusion tensor imaging; FA, fractional anisotropy; HIE, hypoxic-ischemic encephalopathy; ICP, inferior cerebellar peduncle; MCP, middle cerebellar peduncle; MD, mean diffusivity; SCP, superior cerebellar peduncle.

positive correlations between (1) FA values within the thalami and SCP, ICP, and dentate nuclei (Fig. 2a), and (2) MD values within the thalami and MCP, ICP, and dentate nuclei (Fig. 2b). These correlations were most robust for the dentate nuclei.

\section{Discussion}

The human cerebellum has been thought to be resistant to pre- and perinatal HIE. The typical reported patterns of injury in HIE involve the cerebrum and brainstem, and have been extensively described $[6,14]$. Re- ports of cerebellar involvement in HIE are rare [19-21]. Recently, 2 studies revealed a high rate of cerebellar injury in neonatal HIE, using neuropathology and advanced MRI sequences. Aderliesten et al. [15] performed a histopathological analysis of the brains of 23 neonates with HIE who died within 7 days after birth. Activated microglia and macrophages were found in the cerebellar cortex and white matter in the majority (83\%) of cases. Building on these results, Kwan et al. [16] described abnormal DTI measurements, i.e., increased ADC and decreased FA, in the cerebellar peduncles of asphyxiated neonates. Like these studies, we have shown that cerebellar injury, undetectable by qualitative MRI evaluation 


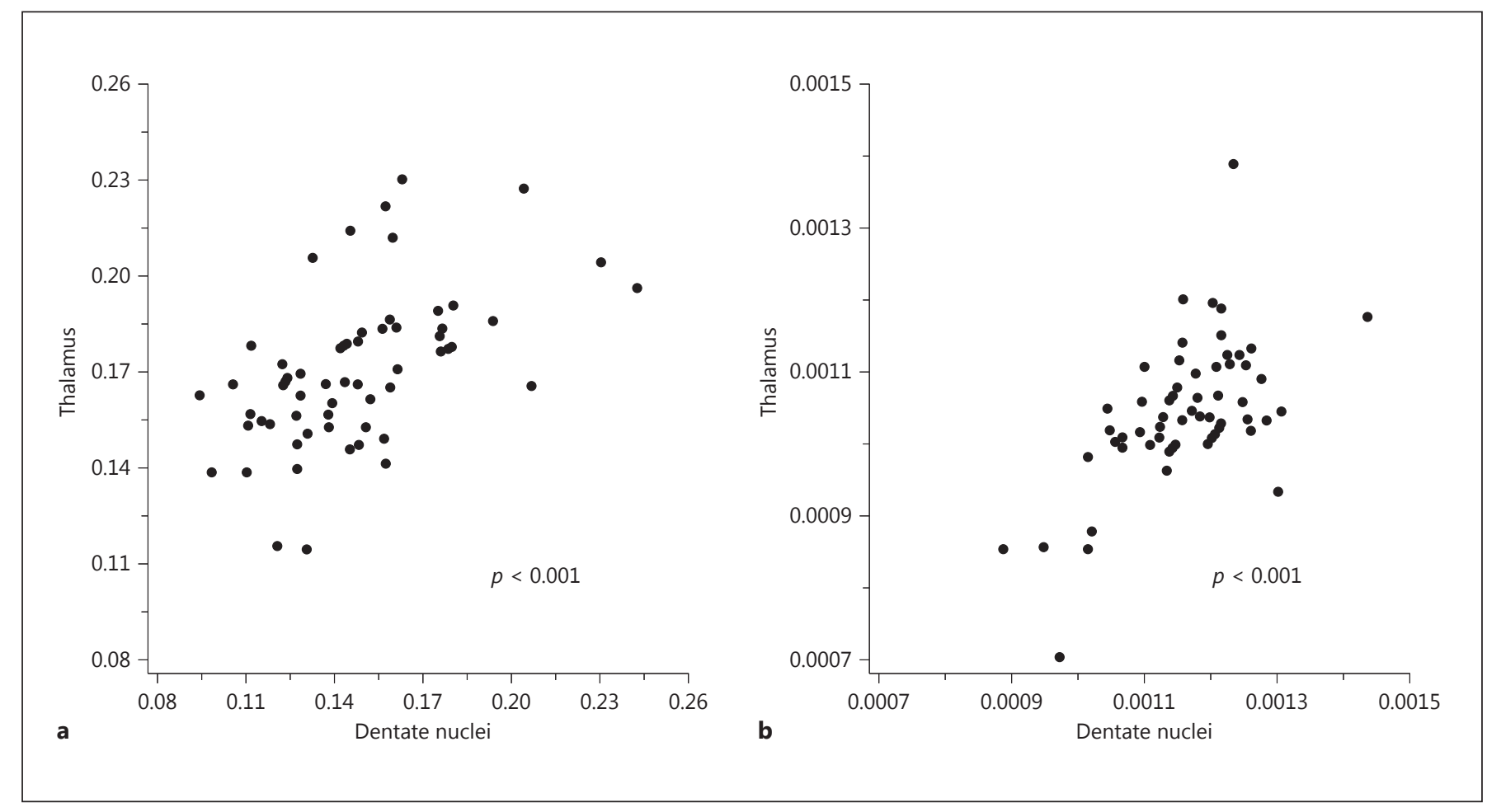

Fig. 2. Scatterplots showing changes in fractional anisotropy (FA) (a) and mean diffusivity (MD) (b) of the dentate nuclei in relation to the thalami. A positive correlation is seen between FA and MD within the dentate nuclei and thalami, respectively.

Table 4. Results of the Spearman rank partial correlation of cerebellar DTI scalars with DTI scalars of thalami in 57 neonates with HIE and hypothermia therapy

\begin{tabular}{lllc}
\hline DTI scalars & $\begin{array}{l}\text { Anatomical } \\
\text { region }\end{array}$ & $\begin{array}{l}\text { Spearman corr. } \\
\text { coefficient }\end{array}$ & $\begin{array}{l}p \\
\text { value }^{\mathrm{a}}\end{array}$ \\
\hline $\mathrm{FA}$ & SCP & 0.33 & $\mathbf{0 . 0 1}$ \\
& MCP & 0.23 & 0.09 \\
& ICP & 0.34 & $\mathbf{0 . 0 1}$ \\
& Dentate nuclei & 0.59 & $<\mathbf{0 . 0 0 1}$ \\
\hline $\mathrm{MD}, \times 10^{-3} \mathrm{~mm} / \mathrm{s}^{2}$ & SCP & 0.26 & 0.06 \\
& MCP & 0.37 & $\mathbf{0 . 0 0 6}$ \\
& ICP & 0.35 & $\mathbf{0 . 0 1}$ \\
& Dentate nuclei & 0.45 & $<\mathbf{0 . 0 0 1}$ \\
& & &
\end{tabular}

Statistically significant correlations are in bold. DTI, diffusion tensor imaging; FA, fractional anisotropy; HIE, hypoxic-ischemic encephalopathy; MD, mean diffusivity.

a Adjusted for gestational week, age at MRI, and MRI category. alone, can be detected using advanced MRI sequences. We additionally show that for patients with severe HIE, DTI metrics differ from those of neonatal control patients.

Cerebellar injury in neonatal HIE has also been shown in animal studies. In piglets, models of perinatal hypoxia demonstrate cerebellar injury [22-24]. In fetal sheep, Purkinje cell death and reduced cerebellar strata occur following hypoxia/asphyxia insults [25]. In mice, reduced cerebellar volumes occurred at postnatal day 18 (p18) in male mice with HIE that were treated with therapeutic hypothermia on p10 [26]. In rats, the data on cerebellar injury in neonatal HIE are mixed. In p16-18 rats, Purkinje cells were shown to be vulnerable to injury and HIE, in part due to increased autophagy [27]. In a slightly lessmature rodent model of HIE, with injury induced at p7, the cerebellum was largely spared $[28,29]$. In animal studies, the presence of cerebellar injury likely depends on the type of animal, its age at injury, and the study design.

Our results show that severe neonatal HIE may affect the cerebellar peduncles. Significant differences in DTI 
scalars were found between the subgroups of neonates with moderate and severe HIE (injury of the basal ganglia/thalamus and/or cortex) and neonatal controls. In addition, FA and MD measurements in the cerebellar peduncles and dentate nuclei showed a positive correlation with thalamic DTI scalars. This suggests a correlation between cerebellar and thalamic injury, as a marker of HIE severity, and that cerebellar injury in HIE is unlikely to occur in isolation. Our results are in agreement with previous work. Sargent et al. [19] showed atrophy of the cerebellar vermis on follow-up MRI studies of patients who had severe acute neonatal HIE with thalamic involvement, while vermian atrophy was not seen in patients who had a prolonged partial asphyxia. Connolly et al. [21] reported an abnormal T2-hyperintense signal in the anterior lobe of the cerebellar vermis in 18 of 30 patients who had acute neonatal HIE, and also a positive correlation between vermian injury and the severity of HIE based on neuroimaging findings and extremely low Apgar scores (0-1). All neonates with cerebellar injury reported by Alderliesten et al. [15] died; these patients had likely suffered from severe neonatal HIE.

In our study, no patients had evidence of cerebellar injury by qualitative ("eye balling") evaluation of conventional MRI sequences and DTI (trace of diffusion and ADC) maps. Quantitative DTI analysis, however, revealed significant differences in DTI scalars of the cerebellar structures when comparing the neonates with severe HIE and the neonatal controls. For the purpose of this study, a systematic qualitative evaluation of the cerebellum was performed by 2 physicians with extensive experience in the field of pediatric neuroimaging. It is unlikely that obvious cerebellar injuries were overlooked. Instead, it is likely that cerebellar injuries were subtle and/ or diffuse and unable to be detected by qualitative evaluation alone. With other conditions, including cerebral palsy, Williams syndrome, and Rett syndrome, quantitative evaluation of DTI data has been shown to detect mild abnormalities that remain undetected by qualitative image evaluation alone [30]. Quantitative, objective DTI scalars can additionally be studied as predictors of motor, cognitive, and behavioral outcome. Quantitative analysis of DTI data should be included in the neuroimaging evaluation of neonates with severe HIE, so as to fully assess the extent of injuries and provide potential predictors of outcome.

This study is limited by its small sample size and warrants confirmation in a larger cohort. Furthermore, while our control cohort was carefully screened for possible neurologic abnormalities, we cannot ensure that all neo-

Cerebellar Injury in Severe Neonatal HIE nates had normal baseline DTI measurements. ROIs were drawn manually, making the analysis observer-dependent; future studies are warranted to ensure our results hold true with automated techniques. More advanced analysis techniques, including neurite orientation dispersion and density imaging, may offer additional insight into the nature of FA changes. Nonetheless, we are confident that none of these limitations impacted our primary result: that cerebellar DTI measures are abnormal in neonates with severe HIE. Patients in our clinical cohort were identified and treated using previous published criteria that have been widely adopted internationally; we expect these results to be generalizable to other clinical cohorts of severely encephalopathic neonates.

In summary, our results suggest that infants with severe HIE may have injury of the neonatal cerebellar white-matter tracts, which appears undetectable by the qualitative analysis of neuroimaging data alone. Cerebellar DTI scalars correlate with thalamic measures, highlighting that cerebellar injury is unlikely to occur in isolation and may be linked to the severity of HIE. The impact of cerebellar injury on long-term neurodevelopmental outcome warrants further study.

\section{Acknowledgment}

We are thankful to Charlamaine M. Parkinson, RNC BSN MS, for her support in the collection of the clinical data for this study.

Monica Lemmon received support from the American Academy of Neurology Medical Education Research Fellowship, is currently supported through the National Palliative Care Research Center, and received compensation for medicolegal work. Kathryn A. Carson's work was supported by the National Center for Research Resources and the National Center for Advancing Translational Sciences (NCATS) of the National Institutes of Health (grant No. 1UL1TR001079). Frances J. Northington's work was supported by the Eunice Kennedy Shriver National Institute of Child Health and Human Development (NICHD) of the National Institutes of Health (grant Nos. HD070996 and HD074593) and Covidien.

\section{Disclosure Statement}

The authors declare that they have no potential conflicts of interest.

Dev Neurosci 2017;39:207-214 


\section{References}

1 Volpe JJ: Neurology of the Newborn, ed 5. Philadelphia, Saunders/Elsevier, 2008.

2 Jacobs SE, Berg M, Hunt R, Tarnow-Mordi WO, Inder TE, Davis PG: Cooling for newborns with hypoxic ischaemic encephalopathy. Cochrane Database Syst Rev 2013: CD003311.

3 Azzopardi D, Strohm B, Marlow N, Brocklehurst P, Deierl A, Eddama O, Goodwin J, Halliday HL, Juszczak E, Kapellou O, Levene M, Linsell L, Omar O, Thoresen M, Tusor N Whitelaw A, Edwards AD, Group TS: Effects of hypothermia for perinatal asphyxia on childhood outcomes. N Engl J Med 2014;371: 140-149.

4 Pappas A, Shankaran S, McDonald SA, Vohr BR, Hintz SR, Ehrenkranz RA, Tyson JE, Yolton K, Das A, Bara R, Hammond J, Higgins RD; Hypothermia Extended Follow-Up Subcommittee of the Eunice Kennedy Shriver NNRN: Cognitive outcomes after neonatal encephalopathy. Pediatrics 2015;135:e624e634.

5 Ferriero DM, Bonifacio SL: The search continues for the elusive biomarkers of neonatal brain injury. J Pediatr 2014;164:438-440.

6 Barkovich AJ, Westmark K, Partridge C, Sola A, Ferriero DM: Perinatal asphyxia: MR findings in the first 10 days. AJNR Am J Neuroradiol 1995; 16:427-438.

7 Takeoka M, Soman TB, Yoshii A, Caviness VS Jr, Gonzalez RG, Grant PE, Krishnamoorthy KS: Diffusion-weighted images in neonatal cerebral hypoxic-ischemic injury. Pediatr Neurol 2002;26:274-281.

8 Chau V, Poskitt KJ, Miller SP: Advanced neuroimaging techniques for the term newborn with encephalopathy. Pediatr Neurol 2009; 40:181-188.

9 Martinez-Biarge M, Diez-Sebastian J, Kapellou O, Gindner D, Allsop JM, Rutherford MA, Cowan FM: Predicting motor outcome and death in term hypoxic-ischemic encephalopathy. Neurology 2011;76:2055-2061.

10 Alderliesten T, de Vries LS, Benders MJ, Koopman C, Groenendaal F: MR imaging and outcome of term neonates with perinatal asphyxia: value of diffusion-weighted MR imaging and (1)H MR spectroscopy. Radiology 2011;261:235-242
11 Rollins N, Booth T, Morriss MC, Sanchez P, Heyne R, Chalak L: Predictive value of neonatal MRI showing no or minor degrees of brain injury after hypothermia. Pediatr Neurol 2014;50:447-451.

12 Thayyil S, Chandrasekaran M, Taylor A, Bainbridge A, Cady EB, Chong WK, Murad S, Omar RZ, Robertson NJ: Cerebral magnetic resonance biomarkers in neonatal encephalopathy: a meta-analysis. Pediatrics 2010;125: e382-e395.

13 van Laerhoven $H$, de Haan TR, Offringa $M$, Post B, van der Lee JH: Prognostic tests in term neonates with hypoxic-ischemic encephalopathy: a systematic review. Pediatrics 2013;131:88-98.

14 Volpe JJ: Neonatal encephalopathy: an inadequate term for hypoxic-ischemic encephalopathy. Ann Neurol 2012;72:156-166.

15 Alderliesten T, Nikkels PG, Benders MJ, de Vries LS, Groenendaal F: Antemortem cranial MRI compared with postmortem histopathologic examination of the brain in term infants with neonatal encephalopathy following perinatal asphyxia. Arch Dis Child Fetal Neonatal Ed 2013;98:F304-F309.

16 Kwan S, Boudes E, Gilbert G, Saint-Martin C, Albrecht S, Shevell M, Wintermark P: Injury to the cerebellum in term asphyxiated newborns treated with hypothermia. AJNR Am J Neuroradiol 2015;36:1542-1549.

17 Shankaran S, Laptook AR, Ehrenkranz RA, Tyson JE, McDonald SA, Donovan EF, Fanaroff AA, Poole WK, Wright LL, Higgins RD, Finer NN, Carlo WA, Duara S, Oh W, Cotten CM, Stevenson DK, Stoll BJ, Lemons JA, Guillet R, Jobe AH; National Institute of Child Health; Human Development Neonatal Research Network: Whole-body hypothermia for neonates with hypoxic-ischemic encephalopathy. N Engl J Med 2005;353:15741584 .

18 Barkovich AJ, Hajnal BL, Vigneron D, Sola A, Partridge JC, Allen F, Ferriero DM: Prediction of neuromotor outcome in perinatal asphyxia: evaluation of MR scoring systems. AJNR Am J Neuroradiol 1998;19:143-149.

19 Sargent MA, Poskitt KJ, Roland EH, Hill A, Hendson G: Cerebellar vermian atrophy after neonatal hypoxic-ischemic encephalopathy. AJNR Am J Neuroradiol 2004;25:1008-1015.

20 Le Strange E, Saeed N, Cowan FM, Edwards $A D$, Rutherford MA: MR imaging quantification of cerebellar growth following hypoxicischemic injury to the neonatal brain. AJNR Am J Neuroradiol 2004;25:463-468.
21 Connolly DJ, Widjaja E, Griffiths PD: Involvement of the anterior lobe of the cerebellar vermis in perinatal profound hypoxia. AJNR Am J Neuroradiol 2007;28:16-19.

22 Thoresen $M$, Haaland $K$, Loberg EM, Whitelaw A, Apricena F, Hanko E, Steen PA: A piglet survival model of posthypoxic encephalopathy. Pediatr Res 1996;40:738-748.

23 Foster KA, Colditz PB, Lingwood BE, Burke C, Dunster KR, Roberts MS: An improved survival model of hypoxia/ischaemia in the piglet suitable for neuroprotection studies. Brain Res 2001;919:122-131.

24 Satas S, Loberg EM, Porter H, Whitelaw A, Steen PA, Thoresen M: Effect of global hypoxia-ischaemia followed by $24 \mathrm{~h}$ of mild hypothermia on organ pathology and biochemistry in a newborn pig survival model. Biol Neonate 2003;83:146-156.

25 Hutton LC, Yan E, Yawno T, Castillo-Melen$\operatorname{dez}$ M, Hirst JJ, Walker DW: Injury of the developing cerebellum: a brief review of the effects of endotoxin and asphyxial challenges in the late gestation sheep fetus. Cerebellum 2014; 13:777-786

26 Burnsed JC, Chavez-Valdez R, Hossain MS, Kesavan K, Martin LJ, Zhang J, Northington FJ: Hypoxia-ischemia and therapeutic hypothermia in the neonatal mouse brain - a longitudinal study. PLoS One 2015;10:e118889.

27 Au AK, Chen Y, Du L, Smith CM, Manole MD, Baltagi SA, Chu CT, Aneja RK, Bayir H, Kochanek PM, Clark RS: Ischemia-induced autophagy contributes to neurodegeneration in cerebellar Purkinje cells in the developing rat brain and in primary cortical neurons in vitro. Biochim Biophys Acta 2015;1852:19021911.

28 Towfighi J, Zec N, Yager J, Housman C, Vannucci RC: Temporal evolution of neuropathologic changes in an immature rat model of cerebral hypoxia: a light microscopic study. Acta Neuropathol 1995;90:375-386.

29 Vannucci RC, Vannucci SJ: A model of perinatal hypoxic-ischemic brain damage. Ann NY Acad Sci 1997;835:234-249.

30 Oishi K, Faria AV, Yoshida S, Chang L, Mori S: Quantitative evaluation of brain development using anatomical MRI and diffusion tensor imaging. Int J Dev Neurosci 2013;31: 512-524. 